\title{
Characterization of surface structure in sputtered Al films: Correlation to microstructure evolution
}

\author{
Adriana E. Lita and John E. Sanchez, Jr. ${ }^{\text {a) }}$ \\ Department of Materials Science and Engineering, University of Michigan, Ann Arbor, Michigan 48109
}

(Received 19 August 1998; accepted for publication 5 October 1998)

\begin{abstract}
Quantitative roughness and microstructural analysis of as-deposited Al films, 0.1-1.0 $\mu \mathrm{m}$ thick, were performed by atomic force microscopy (AFM), one-dimensional power spectral density analysis (1DPSD), transmission electron microscopy, and x-ray pole figure methods. The variation of grain size $(d)$ with thickness $(h)$ in the columnar grained film was $d \propto h^{0.9}$. The initial crystallographic texture was nearly random, with a strong $\mathrm{Al}(111)$ fiber texture evolving by $\approx 0.2$ $\mu \mathrm{m}$ in deposited thickness. AFM imaging revealed a surface structure with hillocks, grains, and grain boundary grooves, and periodic within-grain ridges extending over entire grains. The root-mean-square surface height variation $\left(R_{\mathrm{RMS}}\right)$ initially decreased during deposition but increased as $R_{\mathrm{RMS}} \propto h^{0.55}$ from 0.3 to $1.0 \mu \mathrm{m}$ thickness. The 1DPSD analysis revealed three spatially resolved regimes of roughness evolution; a frequency independent regime at low frequency attributed to hillock growth, an intermediate frequency self-similar regime attributed to grains and grain boundary grooves, and a high frequency self-similar regime attributed to within-grain ridges. Two characteristic dimensions (CD) were defined at the inverse frequencies of transition between each 1DPSD roughness regime. $\mathrm{CD}_{\mathrm{I}}$ at high frequency was identified as the peak-to-peak ridge spacing which remained independent of film thickness. The ridge spacing is proposed to represent the upper limit of an effective surface diffusion length $\left(\lambda_{0}\right)$ due to the effects of surface diffusion and flux shadowing. The $\mathrm{CD}_{\mathrm{II}}$ at lower frequency was identified as the grain size which increased with thickness. The evolution and interactions of roughness and microstructural features are discussed in terms of surface diffusion, grain boundary motion, and flux shadowing mechanisms. (C) 1999 American Institute of Physics. [S0021-8979(99)01502-9]
\end{abstract}

\section{INTRODUCTION}

Deposited polycrystalline thin films have application in a wide variety of magnetic recording and microelectronic devices in which film microstructure and properties often determine the system performance or reliability. As examples, film grain size and texture ${ }^{1}$ control $\mathrm{Al}$ interconnect reliability against electromigration-induced failures, and surface roughness affects the film reflectivity and optical lithography patterning process. The continued development of advanced microelectronic devices therefore requires the careful characterization of surface and microstructural features. However thin film structural details (i.e., grain size, crystallographic texture, and roughness) result from the atomistic processes such as surface diffusion, grain boundary motion, and depositing flux shadowing which operate during film growth. In addition it is possible that different evolutionary regimes of structure development may result from the variation of these atomistic mechanisms with processing conditions. Measurement of surface structure and microstructure as a function of thickness and deposition conditions therefore helps to characterize those regimes of fundamental processes which determine film structure and properties. We present quantitative characterizations of both surface structure and

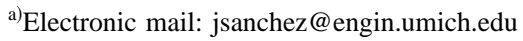

microstructure as well as correlations to surface diffusion, shadowing, and grain growth in sputter deposited Al thin films of varying thickness.

Previous work has described surface structure ${ }^{2-4}$ and microstructure evolution ${ }^{5-8}$ as a function of deposition conditions. Roughness evolution during the deposition of amorphous films was analyzed ${ }^{2,3}$ by modeling surface diffusion and shadowing, while more recent experimental work characterized the quantitative surface structure of polycrystalline $\mathrm{Cu}$ films during growth ${ }^{4}$ without considering correlations to, or the effects from, microstructure evolution. Other work has provided only grain size and texture characterization during film deposition ${ }^{5-8}$ without correlation to detailed surface structure. In one study ${ }^{9}$ the effects of contamination during deposition on average roughness and microstructure were measured only for $0.5-\mu \mathrm{m}$-thick films. The motivation of this work is therefore to provide quantitative characterizations of surface morphology, identify the different regimes of structure development, establish correlations between film surface structure and microstructure evolution, and describe the overall structure development in terms of those processes which act during sputter deposition.

Surface morphology was studied by means of atomic force microscopy (AFM). A computed value of average roughness is the root-mean-square variation (i.e., standard deviation) of the surface height profile from the mean height, $R_{\mathrm{RMS}}$, and is given by 


$$
R_{\mathrm{RMS}}=\left[\frac{1}{N} \sum_{i=1}^{N}\left(y_{i}-\bar{y}\right)^{2}\right]^{1 / 2},
$$

where $N$ is number of data points of the profile, $y_{i}$ are the data points that describe the relative vertical height of the surface, and $\bar{y}$ is the mean height of the surface. The sensitivity or dependence of $R_{\mathrm{RMS}}$ on film thickness $(h)$ is often characterized ${ }^{4}$ by the exponent $(\beta)$ determined from the following relation:

$$
R_{\mathrm{RMS}} \propto h^{\beta} .
$$

The value of the exponent $\beta$ may indicate different growth modes, ${ }^{10}$ however measurement of $\beta$ alone is not sufficient information for growth mode characterization. For example $\beta=0.33$ corresponds to the growth class described by the $\mathrm{KPZ}^{10}$ equation for a one-dimensional interface, and $\beta$ $=0.50$ corresponds to the "random deposition" growth mode. ${ }^{10}$ We note that $R_{\text {RMS }}$ measurements provide only an average measure of surface height variation without regard to possible correlations between specific features and the roughness.

Spatial resolution of the roughness and correlations between roughness and specific surface features are provided by power spectral density ${ }^{11}$ (PSD) analysis. This is accomplished by calculating the square magnitude of the coefficients of the Fourier transform of a digitized surface profile. One- and two-dimensional PSD spectra can be used to characterize the roughness and surface structure. In this work we concentrate on one-dimensional spectra (1DPSD.) For a surface profile line scan the 1DPSD at a given frequency $(f)$ is given by

$$
\operatorname{1DPSD}(f)=\frac{1}{L}\left(\int y(x) e^{i 2 \pi f x} d x\right)^{2},
$$

in units of nanometers cubed, where $L$ is the scan length and $y(x)$ is the line profile. A 1DPSD spectrum provides the variation of PSD power (magnitude) as a function of spatial frequency (or inversely, lateral dimension). The analysis therefore provides the correlation between roughness and the length and spacing of specific surface features. In general, plots with a constant PSD slope indicate consistent surface roughness behavior over a specific frequency range. For example, a flat 1DPSD region (zero slope) indicates a range of frequency independent roughness, whereas a region of finite constant slope indicates self-similar roughness scaling. ${ }^{11}$ The frequency which indicates a transition between two regions of constant 1DPSD slope is defined as $f_{i}$, where the subscript denotes an index if several transitions are found. The $f_{i}$ also defines a "critical dimension" $\left(\mathrm{CD}_{i}\right)$, equal to $1 / f_{i}$, which indicates the length of specific surface features which can be identified by AFM or traditional electron microscopic imaging methods.

The evolution of surface structure, grain size, and crystallographic texture in polycrystalline $\mathrm{Al}$ films ranging from 0.1 to $1 \mu \mathrm{m}$ in thickness has been characterized ${ }^{12}$ in the low substrate temperature regime, $T / T_{m} \leqslant 0.3$, where $T_{m}$ is the $\mathrm{Al}$ melting temperature. In the following we summarize the grain size and crystallographic texture results, provide roughness characterization by $R_{\mathrm{RMS}}$ and 1DPSD analyses, define correlations between the roughness and microstructure, and describe the evolution of film structure in terms of surface diffusion, depositing flux shadowing, and grain boundary motion in the as-deposited $\mathrm{Al}$ films.

\section{EXPERIMENT}

A load locked magnetron sputter system was used to deposit $\mathrm{Al}$ thin films, 0.1 to $1 \mu \mathrm{m}$ thick, onto $150 \mathrm{~mm}$ diam thermally oxidized $\mathrm{Si}$ (100) substrates using high purity Ar at $2 \mathrm{mTorr}$ pressure and at a deposition rate of $1 \mu \mathrm{m} / \mathrm{min}$. Film contamination was minimal since the base pressure of the sputter chamber was less than $\sim 2 \times 10^{-8}$ Torr and wafers were outgassed prior to entering the deposition chamber. Substrates were held at near ambient $\left(\approx 25^{\circ} \mathrm{C}\right)$ temperature during deposition by clamping the substrates to the platen which maintained Ar flow onto the substrate backside. The target diameter is $29.2 \mathrm{~cm}$ and the stationary substrates were centered directly below the target at a spacing of $4.3 \mathrm{~cm}$. This sputtering geometry ensures an even deposition flux across the substrate surface.

Microstructure characterization was performed by a combination of plan view and cross-section transmission electron microscopy (TEM) and x-ray pole figure analysis. TEM sample preparation was carefully controlled to prevent sample heating, and the mean equivalent diameter grain size was obtained via automated image analysis of traced grain boundaries from typically 500-700 grains per thickness. Film crystallographic texture was calculated from a combination of x-ray pole figure analysis and the appropriate thin film defocusing and background corrections. The texture analysis yielded $\mathrm{Al}$ (111) fiber plots ${ }^{12}$ and allowed the calculation of the volume fractions of both the Al (111) fiber oriented and randomly oriented portions at each film thickness. More complete descriptions of film deposition, TEM methods, and x-ray characterizations are provided elsewhere. ${ }^{12}$

Film surface profiles were obtained with an atomic force microscope (Digital Instruments Nanoscope III) operated in tapping mode using an etched single crystal Si tip with a radius of $10 \mathrm{~nm}$. The effects of finite tip error ${ }^{13}$ were not a significant factor in the collection and analysis of the surface profile data, since the ratio of measured surface features to the estimated tip radius varied from 10 to 100 . Collected data consisted of height information on square $512 \times 512$ arrays of pixels which varied from to 2 to $10 \mu \mathrm{m}$ on a side. Lateral resolution (determined as one pixel) was 4 and $20 \mathrm{~nm}$ for the $2 \mu \mathrm{m} \times 2 \mu \mathrm{m}$ and $10 \mu \mathrm{m} \times 10 \mu \mathrm{m}$ scan areas, respectively. The AFM data were used to calculate the $R_{\text {RMS }}$ roughness and 1DPSD spectra for each film thickness. Surface images were obtained from the $2 \mu \mathrm{m} \times 2 \mu \mathrm{m}$ and $5 \mu \mathrm{m} \times 5 \mu \mathrm{m}$ area scans, while the $R_{\mathrm{RMS}}$ surface roughness and 1DPSD spectra were determined from $10 \mu \mathrm{m} \times 10 \mu \mathrm{m}$ scan data. $R_{\mathrm{RMS}}$ was determined by averaging at least ten individual $10 \mu \mathrm{m} \times 10 \mu \mathrm{m}$ scans on randomly located areas for each film thickness. The 1DPSD spectra were obtained by taking the Fourier transform of individual line scans which comprise the area scan, squaring the amplitude of the Fourier coefficients to determine the power, and averaging the (512) 


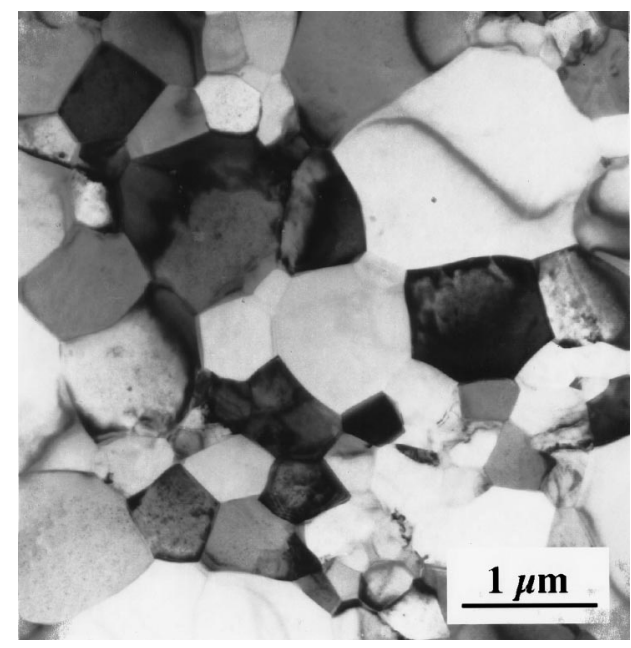

FIG. 1. Plan view transmission electron micrograph of an $0.8-\mu \mathrm{m}$-thick $\mathrm{Al}$ film, showing generally columnar grains. Similar grain structures were found for all film thicknesses.

individual line scan powers at each frequency to generate the 1DPSD. There were no detectable differences in the 1DPSD spectra obtained by averaging the scan lines in the fast scan (horizontal) or slow scan (vertical) directions. The data presented here are for 1DPSD averaged in the fast scan direction. In addition, no in plane directionality in microstructure or surface structure was observed. A $10 \mu \mathrm{m} \times 10 \mu \mathrm{m}$ scan was found to encompass the appropriate range of length scales, i.e., larger than the grain size of approximately $1 \mu \mathrm{m}$ for the thickest film, yet with sufficient resolution to allow for identification of the smaller features. The AFM images were processed using second order plane fitting and second order flattening routines. The leveling routines were applied in order to remove the $z$ offset between scan lines and the tilt and bow in each scan line. All AFM images were processed using the same leveling procedure, with the final images indicating a flat planar profile, as expected. These procedures did not significantly affect the magnitude or shape of the power spectrum or change the characteristic dimensions extracted from the 1DPSD spectra.

TABLE I. The equivalent diameter mean grain size and Al (111) crystallographic texture as a function of film thickness for pure $\mathrm{Al}$ sputter deposited thin films.

\begin{tabular}{ccc}
\hline \hline $\begin{array}{c}\text { Thickness } \\
h(\mu \mathrm{m})\end{array}$ & $\begin{array}{c}\text { Mean grain size } \\
d(\mu \mathrm{m})\end{array}$ & $\begin{array}{c}\text { Al (111) texture } \\
(\text { volume fraction })\end{array}$ \\
\hline 0.1 & 0.11 & 0.53 \\
0.2 & 0.23 & 0.80 \\
0.3 & 0.27 & 0.85 \\
0.4 & 0.36 & 0.87 \\
0.5 & 0.37 & 0.90 \\
0.6 & 0.50 & 0.91 \\
0.7 & 0.56 & 0.92 \\
0.8 & 0.69 & 0.92 \\
0.9 & 0.73 & 0.93 \\
1.0 & 0.91 & 0.93 \\
\hline \hline
\end{tabular}
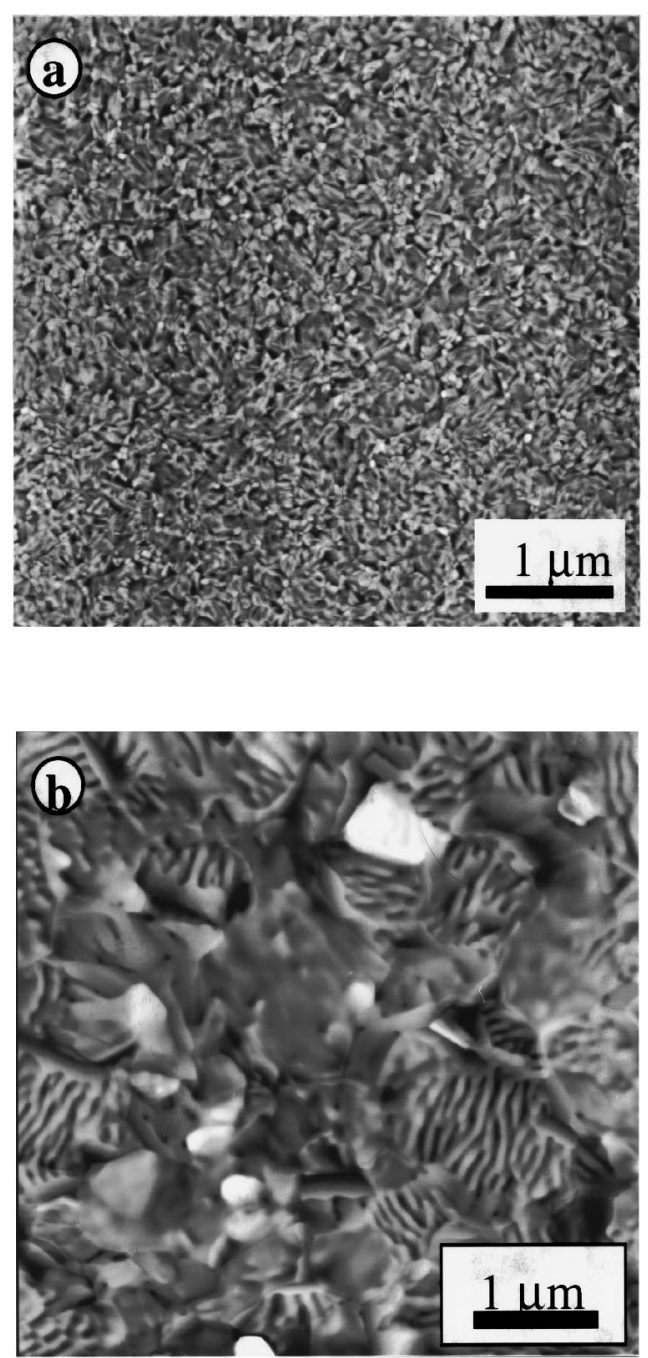

FIG. 2. AFM images of $5 \mu \mathrm{m} \times 5 \mu \mathrm{m}$ areas of (a) an $0.1 \mu \mathrm{m} \mathrm{Al} \mathrm{film,}$ showing the uniformly fine roughness, and (b) an $0.8 \mu \mathrm{m} \mathrm{Al} \mathrm{film,} \mathrm{showing}$ the large hillocks, grains and grain boundary grooves, and within-grain ridges.

\section{RESULTS}

\section{A. Microstructure}

TEM analysis revealed the grain morphology to be primarily columnar for all films, Fig. 1. Grain size distributions were monomodal and lognormal for all film thicknesses. The mean grain size $(d)$ varied with film thickness $(h)$, as $d$ $\approx h^{0.9}$. This dependence coupled with the consistent columnar grain structure indicates that the grain growth was accomplished by grain boundary motion rather than competitive adatom incorporation at the growing film surface. ${ }^{12}$

$\mathrm{X}$-ray pole figure analysis revealed the initial Al film texture to be comprised of both random and (111) fiber texture components. The strong (111) fiber texture development is accomplished in the early stages of growth ${ }^{12}$ when the film evolves from nearly randomly oriented (at $0.1 \mu \mathrm{m}$ thickness) to $\approx 80 \%$ of the film volume (111) fiber oriented (at $0.2 \mu \mathrm{m}$ thickness). Thereafter only gradual textural improvements are possible as deposition proceeds up to $1.0 \mu \mathrm{m}$ thickness. The grain size and $\mathrm{Al}$ (111) texture results are listed in Table I. 


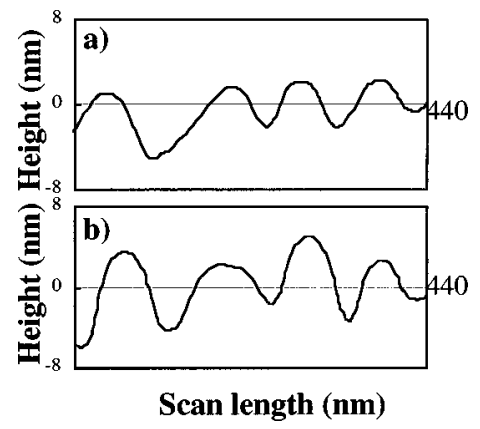

FIG. 3. Cross-section line profiles of the ridge structures for the (a) $0.5 \mu \mathrm{m}$ and (b) $1.0 \mu \mathrm{m} \mathrm{Al} \mathrm{films.} \mathrm{Note} \mathrm{the} \mathrm{relatively} \mathrm{constant} \mathrm{wavelength} \mathrm{but} \mathrm{in-}$ creasing height of the ridges with thickness.

\section{B. Surface structure}

The $5 \mu \mathrm{m} \times 5 \mu \mathrm{m}$ AFM topographs of Al films revealed a varied surface morphology (Fig. 2). The different features can be identified as hillocks scattered randomly at average separations larger than the grain size, obvious grains and grain boundary grooves, and periodic ridge features with spacing smaller than the grain size but which extend uniformly over the surface of individual grains. Similar surface morphologies were found on all $\mathrm{Al}$ films thicker than 0.3 $\mu \mathrm{m}$, with the ridge and hillock features missing below this thickness, Fig. 2(a).

Cross-section profiles of the ridge structures, Fig. 3, indicate that they grow in height but maintain a nearly constant periodicity during continued deposition. The ridge spacing is determined from measurement of the average peak-to-peak distance for each film thickness. The average ridge spacing is approximately constant during film deposition from $\approx 0.3$ to $1.0 \mu \mathrm{m}$.

The $R_{\mathrm{RMS}}$ values suggest relatively smooth surfaces and indicate two regimes of $R_{\mathrm{RMS}}$ variation with thickness. $R_{\mathrm{RMS}}$ is nearly constant or slightly decreasing for films less than $0.4 \mu \mathrm{m}$ thick but monotonically increases with thickness thereafter (Fig. 4). The error bars in Fig. 4 result from the averaging of $\approx 10$ scans. The overall $R_{\mathrm{RMS}}$ dependence on film thickness [Eq. (2)] yields $\beta=0.33$, however if only $R_{\mathrm{RMS}}$ data from films equal to and greater than $0.3 \mu \mathrm{m}$ are analyzed, $\beta=0.55$. A similar analysis ${ }^{4}$ for as-deposited $\mathrm{Cu}$

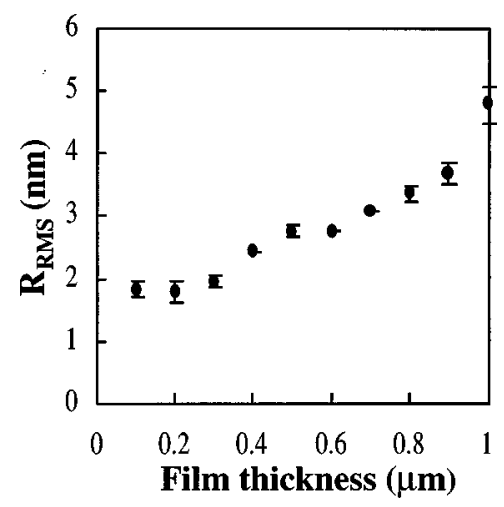

FIG. 4. $R_{\text {RMS }}$ average roughness determined from AFM scans as a function of film thickness. The $R_{\text {RMS }}$ initially decreases during the early stages of the film formation but increases as $R_{\mathrm{RMS}} \alpha h^{0.55}$ from $\approx 0.3$ to $1.0 \mu \mathrm{m}$.

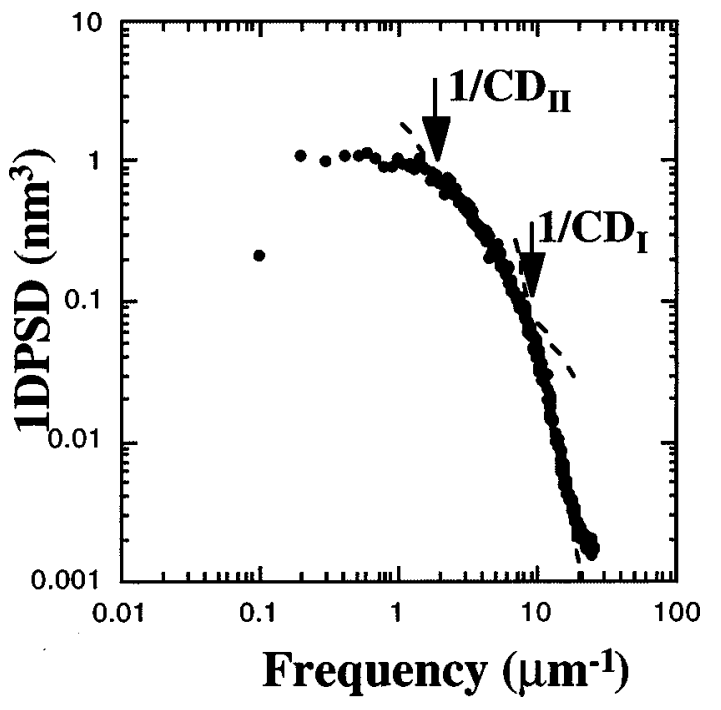

FIG. 5. 1DPSD spectrum for the $0.6 \mu \mathrm{m} \mathrm{Al} \mathrm{film,} \mathrm{illustrating} \mathrm{the} \mathrm{three}$ regimes of roughness; frequency independent roughness at low frequency associated with hillocks, and the two self-similar constant slope roughness regimes at intermediate and high frequency. The transition frequencies between the roughness regimes define the characteristic dimensions $C_{\mathrm{I}}$ and $\mathrm{CD}_{\mathrm{II}}$, which represent the ridge spacing and grain size, respectively.

films yielded $\beta=0.33$, similar to the overall $R_{\mathrm{RMS}}$ behavior observed here. However that previous analysis did not find the two regimes of $R_{\text {RMS }}$ variation found in here, Fig. 4, possibly because of the greater resolution in film thickness. We note that $\beta=0.55$ corresponds to a random deposition mode classification. ${ }^{10}$

A typical 1DPSD spectrum, Fig. 5, illustrates frequencyindependent roughness at long length scales (low frequency), with frequency-dependent (constant slope) 1DPSD magnitude at smaller length scales indicating self-similar roughness at these higher frequencies. In addition films $0.3 \mu \mathrm{m}$ and thicker have two separate regions of self-similar constant slope PSD behavior, Fig. 5. The 1DPSD spectra have the same form for all thicknesses greater than $0.2 \mu \mathrm{m}$, indicating generally similar behavior as film deposition proceeds.

The frequency independent 1DPSD roughness at low frequency increases with thickness, Fig. 6. Given that the range of length scale associated with this roughness is larger than $1 \mu \mathrm{m}$, we ascribe this roughness to hillocks, evident in Fig. 2, which are randomly situated and separated by several grain diameters, and which obviously increase in height during deposition.

The 1DPSD magnitude at intermediate frequencies also increases monotonically with thickness, Fig. 6. Conversely, at the highest frequencies the 1DPSD magnitude initially decreases with thickness (below $0.3 \mu \mathrm{m}$ thick) and subsequently remains nominally constant with thickness. The transition from high to intermediate frequency self-similar roughness behavior, which defines $\mathrm{CD}_{\mathrm{I}}$, is approximately constant with thickness which indicates a surface feature with constant dimension. Note that the length associated with the $\mathrm{CD}_{\mathrm{I}}$ is approximately equal to the measured ridge spacing for each thickness, Fig. 7, which is also constant with 


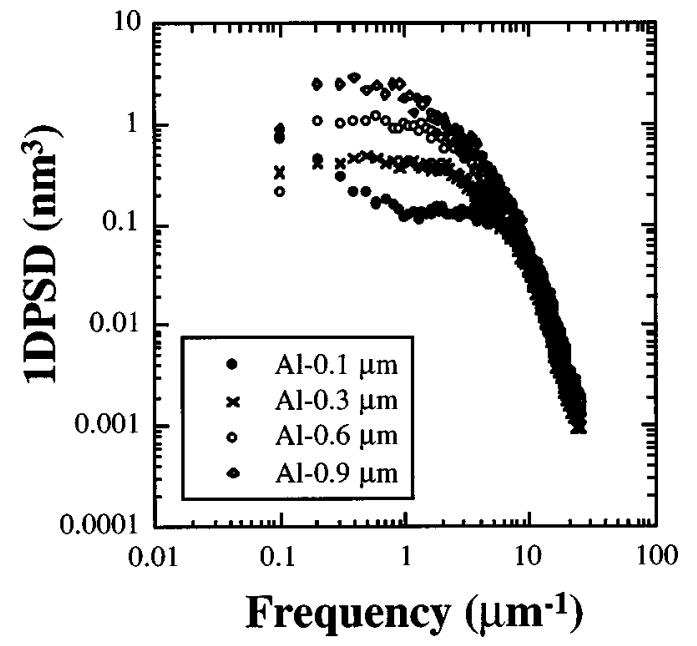

FIG. 6. 1DPSD spectra for the 0.1-, 0.3-, 0.6-, and 0.9- $\mu$ m-thick Al films, illustrating the generally increasing roughness at low and intermediate frequencies with further deposition.

thickness. We therefore associate the $\mathrm{CD}_{\mathrm{I}}$ with the withingrain ridge spacing.

Similarly, the transition point between the 1DPSD low frequency roughness and the intermediate frequency selfsimilar roughness defines a characteristic length, $\mathrm{CD}_{\mathrm{II}}$, for each thickness. We note that $\mathrm{CD}_{\mathrm{II}}$ and the mean grain size are approximately equal and vary similarly with thickness, Fig. 8. We therefore identify $\mathrm{CD}_{\mathrm{II}}$ as the mean grain size, $d$. The correlations of $\mathrm{CD}_{\mathrm{I}}$ and $\mathrm{CD}_{\mathrm{II}}$ to the ridge spacing and grain size, respectively, and their significance with respect to atomistic processes operating during film formation are discussed below.

\section{DISCUSSION}

The effects of grain size evolution during film deposition are evident on the variation of crystallographic texture and the $R_{\mathrm{RMS}}$ roughness with thickness. Grain growth leads to the rapid development of a primarily $\mathrm{Al}$ (111) fiber oriented film early in the deposition, where the grain boundary motion is driven by curvature, surface, and interface energy minimization forces. ${ }^{14}$ Surface energy minimization may lead to the abnormal grain growth process ${ }^{14}$ which is often

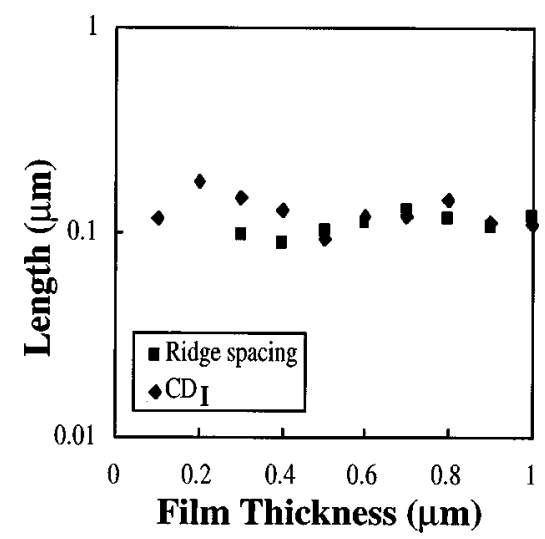

FIG. 7. Correlation between the measured ridge spacing and the $\mathrm{CD}_{\mathrm{I}}$ (defined only for films $0.3 \mu \mathrm{m}$ and thicker) during film deposition.

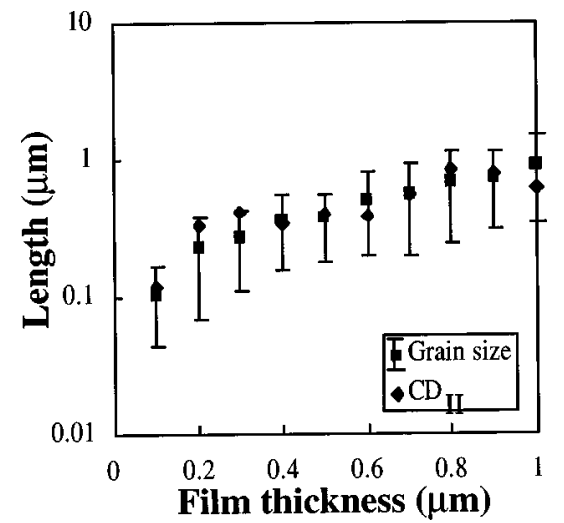

FIG. 8. Correlation between the measured equivalent diameter mean grain size and $\mathrm{CD}_{\mathrm{II}}$ which indicates that the grain size is the lower length scale limit for the hillock roughness.

distinguished by a bimodal grain size distribution. While the strong $\mathrm{Al}$ (111) texture development is an obvious result of surface and interfacial energy minimization, only monomodal grain distributions were observed in the present results. Also note that the development of a primary Al (111) fiber texture from an initial randomly oriented film ${ }^{15}$ was previous found in as-evaporated $\mathrm{Al}$ films.

During the early stages of deposition, proportionately larger portions of the film volume ${ }^{12}$ are swept by migrating boundaries which results in efficient Al (111) texture development. During this stage obviously many grains are eliminated along with the contributions of grain surface structure and boundary grooves to the overall roughness. Therefore the total roughness $\left(R_{\mathrm{RMS}}\right)$ decrease is a primary consequence of the grain growth process during deposition up to $\approx 0.3 \mu \mathrm{m}$ in thickness. However as the film thickens relatively smaller portions of the film are swept by migrating boundaries, allowing roughening processes such as hillock growth and shadowing to increase the total film roughness, $R_{\text {RMS }}$, Fig. 4. The contributions of surface diffusion to the initial $R_{\text {RMS }}$ decrease may be significant and will be discussed below:

As noted earlier, the ridges maintain a consistent direction within each grain, suggesting that they indicate a specific crystallographic direction. Initial characterization has not revealed a conclusive in-plane alignment of the ridges. Given the predominant $\mathrm{Al}$ (111) fiber texture even (111) oriented grains are observed to maintain a ridge surface structure during deposition. Similar ridges in as-deposited Al films have been previously observed, ${ }^{15}$ however no descriptions of explanation for their formation were provided. We propose that the ridges develop in order to provide ledge sites which can accommodate the rapid adatom incorporation into the (111) surfaces ${ }^{12}$ which normally maintain a low defect density. ${ }^{16}$ A similar mechanism has been used to explain the rapid development of $\approx 5^{\circ}$ misalignment of growing $\mathrm{Si}$ (111) surfaces during high rate epitaxial growth. ${ }^{17}$

The rates of roughness evolution for the three spatially resolved regimes can be illustrated by plotting the 1DPSD magnitude at specific length scales (i.e., inverse frequency) as a function of thickness, Fig. 9. The low frequency "hillock" roughness increases with thickness due to shadowing, 


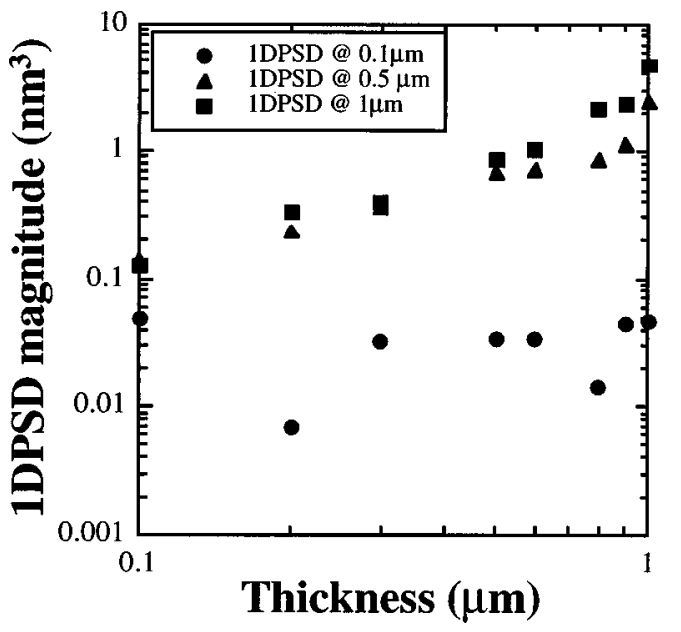

FIG. 9. Variation of the 1DPSD magnitude in the three length regimes; the low frequency hillock roughness is measured at $(1.0 \mu \mathrm{m})^{-1}$, the intermediate frequency is measured at $(0.5 \mu \mathrm{m})^{-1}$, and the high frequency roughness is measured at $(0.1 \mu \mathrm{m})^{-1}$.

resulting in hillock growth at a rate exceeding the average deposition rate. We assume that surface diffusion cannot effectively reduce the surface curvature at this length scale under these deposition conditions. The mean grain size places a lower limit on the size and spacing of hillocks, since hillocks are simply individual grains of height significantly greater than the film thickness. Therefore frequency limit for the hillock roughness is $\approx 1 / d$. However since the low frequency roughness upper frequency limit was previously defined as $\left(1 / \mathrm{CD}_{\mathrm{II}}\right)$, we simply identify $\mathrm{CD}_{\mathrm{II}}$ as the mean grain size, $d$.

The high frequency 1DPSD magnitude decrease with thickness, Fig. 9, is also attributed to surface diffusion which acts to reduce surface curvature. Note that the average ridge height increases during deposition, Fig. 3, while the ridge spacing remains constant, indicating that the ridge spacing is greater than can be significantly reduced by surface diffusion. The ridge spacing thus serves as the upper limit of surface length (or equivalently, lower limit of spatial frequency) for the smoothing of surface profiles by diffusion along the surface.

Previous analysis ${ }^{2,18}$ examined the stability of surface perturbations against the smoothing effects of surface diffusion. Srolovitz, Mazor, and Bukiet ${ }^{2}$ defined a temperaturedependent effective surface diffusion length, $\lambda_{0}$, such that surface wavelengths less than $\lambda_{0}$ are smoothed by diffusion while longer wavelength perturbations continue to grow in height during deposition. $\lambda_{0}$ is defined as $\lambda_{0}$ $=\left(4 \pi^{2} D_{s} \sigma_{s} \Omega^{2} \epsilon / K_{b} T \delta J\right)^{1 / 2}$, where $D_{s}=$ surface diffusivity, $\sigma_{s}=$ specific surface energy, $\Omega=$ atomic volume, $\epsilon$ = number of atoms per unit area, $K_{b} T=$ thermal energy, $\delta$ $=$ atomic radius, and $J=$ volume rate of deposition flux arriving per unit area. The surface diffusivity and activation energy may be estimated by equating the ridge spacing and the above expression for $\lambda_{0}$. Using the appropriate values for $\mathrm{Al}$ and the $300 \mathrm{~K}$ and $1 \mu \mathrm{m} / \mathrm{min}$ deposition conditions results in a surface diffusivity $D_{s}=5.7 \times 10^{-12} \mathrm{~cm}^{2} / \mathrm{sec}$. Further, assuming the diffusion pre-exponential $D_{0}$ factor to be
$10^{-2}$ or $10^{-4} \mathrm{~cm}^{2} / \mathrm{sec}$ yields a surface diffusion activation energy value of 0.43 and $0.55 \mathrm{eV}$, respectively, similar to values reported elsewhere. ${ }^{16}$ As shown above the roughness at length scales smaller than the ridge spacing decreases with deposition, while the ridges increase in height. Thus it is reasonable to conclude that the stable ridge spacing represents the upper limit of the surface diffusion length, $\lambda_{0}$, for $\mathrm{Al}$ surfaces under these deposition conditions. We also conclude that surface diffusion contributes to the $R_{\mathrm{RMS}}$ decrease in the early stages of film deposition, since the grain size in this early regime is $\approx 0.1 \mu \mathrm{m}$ and is in the range of $\lambda_{0}$.

The combined action of shadowing and surface diffusion during the deposition of columnar grained thin films was previously analyzed ${ }^{2,4}$ for films deposited at similar temperatures, $T / T_{m} \leqslant 0.3$. We have extended those characterizations by combining grain size and texture measurements with quantitative surface analysis, and by distinguishing the spatially resolved regimes of thin film structure development. This analysis illustrates the interactions between surface structure and microstructure evolution for sputter deposited Al thin films under these deposition conditions, and allows the identification of the processes such as grain growth and surface diffusion which control different regimes of structure evolution during deposition.

\section{CONCLUSIONS}

The evolution of surface morphology and microstructure of sputter deposited polycrystalline Al films deposited on amorphous substrates was characterized by TEM, $\mathrm{x}$-ray, and AFM techniques. Al grain morphology remained columnar during film growth, with the mean grain size increasing with thickness as $d \alpha h^{0.9}$. A primary $\mathrm{Al}$ (111) texture component, centered $\approx 5^{\circ}$ from exact fiber orientation, evolved from a nearly randomly oriented $0.1-\mu \mathrm{m}$-thick film into a well (111) textured $0.2-\mu \mathrm{m}$-thick film [0.80 Al (111) volume fraction], with gradual sharpening of the $\mathrm{Al}$ (111) texture during further deposition.

For the thinnest films the surface structure illustrated by AFM images shows uniformly rough features. The $R_{\text {RMS }}$ average roughness decreased during deposition in this early regime, from 0.1 to $0.3 \mu \mathrm{m}$, which was attributed to the effects of grain growth and surface diffusion. Film surfaces thereafter are comprised of widely separated hillocks, grains and boundary grooves, and within-grain ridges. Accordingly the $R_{\mathrm{RMS}}$ increased with deposition as $\beta=0.55$ for thicknesses beyond $0.3 \mu \mathrm{m}$. Although grain growth is still occurring, this roughening is due to shadowing of the arriving atomic flux leading to hillock growth and the increase in ridge height.

Quantitative surface characterization by spatially resolved 1DPSD analysis identified three regimes of roughness evolution during film growth. At large wavelength (low frequency) the surface was characterized by frequencyindependent roughness which increases with thickness and is attributed to the growth of randomly scattered hillocks due to flux shadowing. At smaller wavelengths (higher frequencies) the 1DPSD spectra are characterized by two self-similar regions of constant slope. At intermediate frequencies the 
1DPSD magnitude also increases with thickness although at a lower rate than the frequency-independent roughness. At the highest frequencies the 1DPSD magnitude initially decreases then remains generally constant with thickness due to the effects of surface diffusion.

The $\mathrm{CD}_{\mathrm{I}}$ defined as the inverse transition frequency between high and intermediate frequency 1DPSD roughness, is identified as the within-grain ridge spacing $(\approx 0.1 \mu \mathrm{m})$ which remains generally constant with thickness. The ridge spacing is also interpreted as somewhat greater than the diffusion length, $\lambda_{0}$, as described in other analytical work. The $\mathrm{CD}_{\mathrm{II}}$, defined as the inverse transition frequency between the intermediate and low frequency 1DPSD magnitude, is identified as the mean grain size which increases with thickness.

Ongoing work is concerned with determination of the crystallographic direction of the within-grain surface ridges, and the extension of similar surface-microstructure characterizations to deposited $\mathrm{Cu}$ films. Future work will address the effects of substrate material, deposition temperature, and alloying additions on the processes which drive microstructure evolution in thin films.

\section{ACKNOWLEDGMENTS}

The authors acknowledge the assistance of R. Cutler (Univ. Michigan) with grain size analysis, C. Wauchope
(Univ. Michigan) with X-ray analysis and TEM, S. DuPrey (Univ. Michigan, now at Motorola Corporation) with TEM sample preparation, and J. Barragan (Advanced Micro Devices) with $\mathrm{Al}$ depositions.

${ }^{1}$ S. Vaidya and A. K. Sinha, Thin Solid Films 75, 253 (1981).

${ }^{2}$ D. J. Srolovitz, A. Mazor, and B. G. Bukiet, J. Vac. Sci. Technol. A 6, 2371 (1988)

${ }^{3}$ G. S. Bales and A. Zangwill, J. Vac. Sci. Technol. A 9, 145 (1991).

${ }^{4}$ C. Eisenmenger-Sittner, A. Bergauer, and H. Bangert, J. Appl. Phys. 78, 4899 (1995).

${ }^{5}$ B. A. Movchan and A. V. Demchishin, Fiz. Met. Metalloved. 28, 653 (1969).

${ }^{6}$ J. A. Thornton, Annu. Rev. Mater. Sci. 7, 239 (1977).

${ }^{7}$ C. R. M. Grovenor, H. T. G. Hentzell, and D. A. Smith, Acta Metall. 32, 773 (1984).

${ }^{8}$ E. Grantscharova, Thin Solid Films 224, 28 (1993).

${ }^{9}$ M. J. Verkerk and W. Brankaert, Thin Solid Films 135, 77 (1986).

${ }^{10}$ A. Barabasi and H. E. Stanley, Fractal Concepts in Surface Growth (Cambridge University Press, Cambridge, 1995).

${ }^{11}$ J. M. Elson and J. M. Bennett, Appl. Opt. 34, 201 (1995).

${ }^{12}$ J. E. Sanchez, Jr., A. Lita, and C. Wauchope (unpublished).

${ }^{13}$ K. L. Westra and D. J. Thomson, J. Vac. Sci. Technol. B 13, 344 (1995).

${ }^{14}$ C. V. Thompson, J. Appl. Phys. 58, 763 (1985).

${ }^{15}$ S. Roberts and P. J. Dobson, Thin Solid Films 135, 137 (1986).

${ }^{16}$ R. Stumpf and M. Scheffler, Phys. Rev. Lett. 72, 254 (1994).

${ }^{17}$ S. M. Sze, VLSI Technology (McGraw-Hill, New York, 1983), p. 69.

${ }^{18}$ W. W. Mullins, J. Appl. Phys. 28, 333 (1957). 